Archive for

Organic Chemistry

Arkivoc 2020, part viii, 125-135

\title{
Microwave induced synthesis of a new class of pyrano isoxazoline and isoxazole annulated chromones - an intramolecular nitrile oxide cycloaddition with tethered olefins and alkynes
}

\begin{abstract}
Jayaprakash Rao Yerrabelly, ${ }^{* a}$ Shyam Prasad Nuligonda, ${ }^{a}$ Sudhakar Mokenapelli, ${ }^{a}$ and Prasad Rao Chitneni ${ }^{\mathrm{a}}$
${ }^{a}$ Natural Products Laboratory, Department of Chemistry, Osmania University, Hyderabad-500007, Telangana, India
\end{abstract}

Email: yjpr19@yahoo.com

Received 05-03-2020

Accepted 11-02-2020

Published on line $12-11-2020$

\section{Abstract}

A variety of new highly substituted 6-6-6-5-membered tetracyclic pyrano isoxazoline/isoxazole annulated chromone derivatives have been synthesized via eco-friendly microwave assisted/ceric ammonium nitrate (CAN) as an oxidant, intramolecular 1,3-dipolar cycloaddition with in situ generated nitrile oxides from aldoximes of alkene/alkyne tethered chromones. This protocol is practically simple and efficient to construct diverse range of substituted pyrano isoxazoline/isoxazole annulated chromone derivatives and gave higher yields of products in microwave irradiation compared to conventional heating. The structures of all the synthesized compounds were established by IR, NMR and MASS spectral analysis.<smiles></smiles><smiles>[R]c1cc2c(=O)c(C)c(C)oc2c(C)c1O</smiles><smiles>[R]c1cc2c(=O)c(C)c(C)oc2c2c1OCC1C2=NOC1([R])[R]</smiles>

a: One pot synthesis of allylation and aldoxime b: One pot synthesis propargylation and aldoxime

c: CAN mediated microwave assisted intramolecular 1,3-dipolar cycloaddition

Keywords: Chromone aldoximes, nitrile oxides, 1,3-dipolar cycloaddition, pyrano isoxazoline/isoxazole 


\section{Introduction}

Chromone heterocyclic frameworks are privileged scaffolds widely occur in the natural products especially in plant kingdom. ${ }^{1,2}$ Chromone derivatives extensively showed diverse biological activities ${ }^{3-8}$ such as antiinflammatory, anti-viral, antioxidant, anti-tumor, anti-hypertensive and also proved as Tyrosine kinase protein inhibitors. Isoxazolines are found to present in wide range of biologically active compounds. Mainly, chromeno[4,3-c]isoxazolines ${ }^{9}$ exhibits anti-psychotic, anti-depressant and anti-anxiety activities. Because of labile $\mathrm{N}-\mathrm{O}$ group of isoxazoline are the rich source of desired bi-functional group ${ }^{10}$ like 1,3 -amino alcohols, $\beta$ hydroxy ketones, $\beta$-hydroxy nitriles, unsaturated oximes and also versatile intermediates. Additionally, isoxazoles are the five membered nitrogen, oxygen heterocyclics act as lysophosphatidic acid (LPA) antagonists, ${ }^{11}$ inhibitors of human rhinovirus-2-replication, ${ }^{12}$ insect anti-feedant, ${ }^{13}$ anti-tubulin, ${ }^{14}$ selective agonists of dopamine D4 receptors, ${ }^{15}$ GABA antagonist, ${ }^{16}$ COX-2 inhibitory ${ }^{17,18}$ and anti-cancer agents. ${ }^{19-21}$ Isoxazoline and Isoxazole were also applied as dyes, electric insulating oils and high temperature lubricants ${ }^{22}$, ${ }^{23}$ and also as synthetic precursor of bioactive natural products ${ }^{24}$ (Figure 1 ).

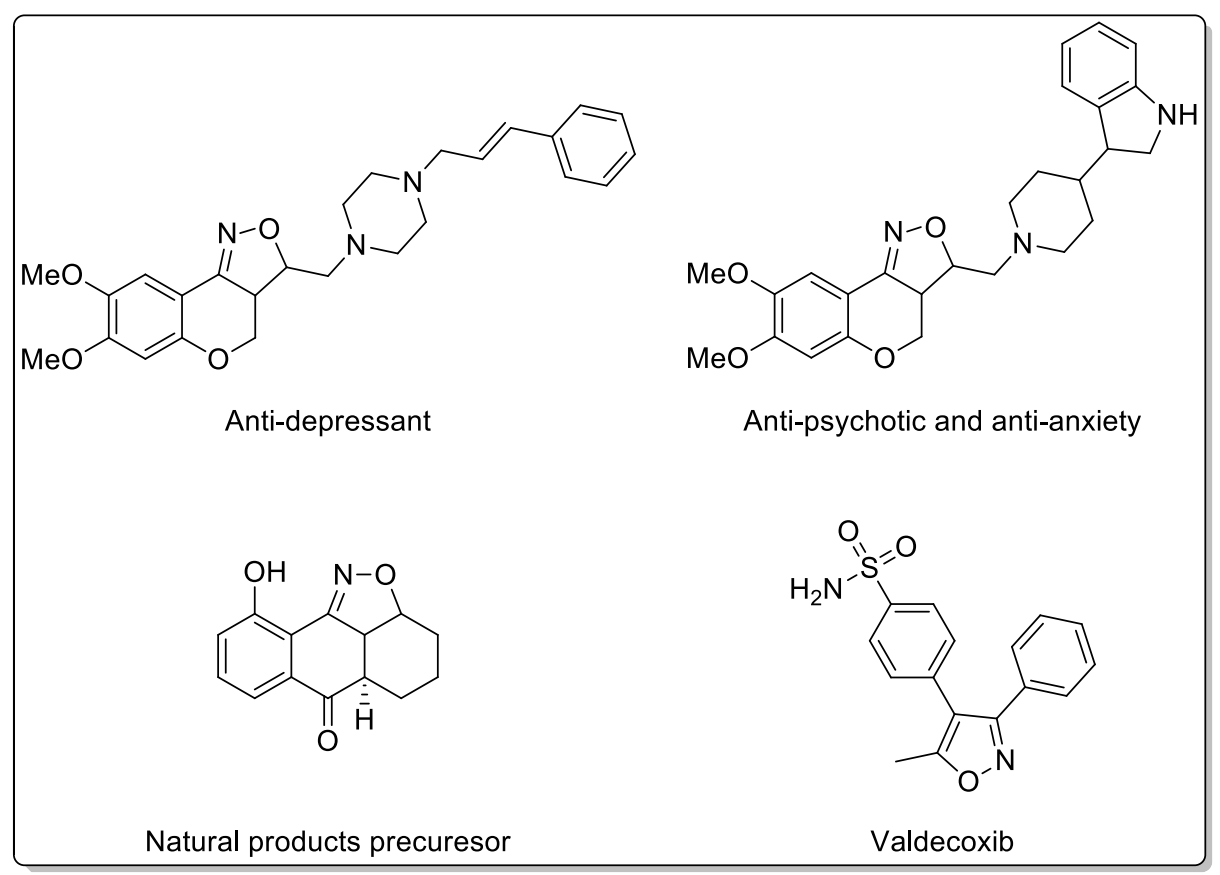

Figure 1. Biologically active isoxazoline/ isoxazole derivatives.

The intramolecular nitrile oxide 1,3-dipolar cycloaddition is the dominant methodology to construct complex polycyclic rings in a single step reaction with an excellent regio/stereo selectivity. ${ }^{25-29}$ In view of the wide biological activities exhibited by the chromones/isoxazolines/isoxazoles and our interest in developing biologically active heterocyclic ring fused chromone derivatives, we planned for the design and synthesis of novel tetracyclic pyrano isoxazoline/isoxazole annulated chromone derivatives using intramolecular $[1,3]$ dipolar cycloaddition of nitrile oxides. Usually, alkenes and alkynes are the good dipolarophiles for the 1,3dipolar cycloaddition with nitrile oxides to produce directly bicyclic pyrano isoxazoline/isoxazoles. Herein, we report the synthesis of tetracyclic 6-6-6-5-membered pyrano isoxazoline/isoxazole annulated chromones by tandem intramolecular 1,3-dipolar cycloaddition of in situ generated nitrile oxides from alkene/alkyne tethered chromone aldoximes using conventional as well as microwave induced methods. Microwave induced 
organic synthesis is emerged as powerful eco-friendly method in developing diverse range of biologically potential heterocyclic compounds in drug discovery program, because of its significant role in improved yields of products, short reaction time, minimum wastage, maximum atom economy and method to approach green synthesis. $^{30}$

\section{Results and Discussion}

7-hydroxy-8-formyl-2,3-dimethylchromones $\mathbf{1 a - b}$ were synthesized using our earlier reported procedure. ${ }^{31}$ The key intermediates, alkene appended chromone-8-aldoxime derivatives $\mathbf{2 a - h}$ were prepared in one pot by treating the bifunctional hydroxy aldehydes $\mathbf{1} \mathbf{a}-\mathbf{b}$ with substituted allyl bromides, hydroxylamine hydrochloride, in the presence of sodium acetate in DMF at $70{ }^{\circ} \mathrm{C}$. The allylation and hydroxylamine hydrochloride condensation with aldehyde at one time in a single step smoothly proceeded to furnish products $\mathbf{2 a - h}$ in good yields. By the routine method these are prepared in two separate steps, initially allylation at phenolic function followed by condensation of hydroxylamine hydrochloride with aldehyde. A number of oxidants and Lewis catalysts were evaluated using suitable solvents for the in situ generation of nitrile oxide from the aldoxime intermediate $\mathbf{2 a}$ (Table-1) followed by dipolar cycloaddition at alkene to afford pyrano isoxazoline annulated chromones 3a. Among the oxidants and catalysts employed, we found to furnish CAN (0.002 mol) mediated intramolecular nitrile oxide addition product 3a in a little higher yield (60\%) compared to all other variants. Under these optimized conditions the derivatives $\mathbf{3 b}$-h were prepared from their corresponding substrates (Scheme-1).

Table 1. Optimization reaction conditions for the synthesis of compound 3a using various catalysts/oxidants

\begin{tabular}{cccccc}
\hline Entry & Catalysts/Oxidants $^{\text {a }}$ & Solvent $^{\mathrm{b}}$ & ${\text { Temp }\left({ }^{\circ} \mathrm{C}\right)}$ & Time $(\mathrm{h})$ & Yield $^{\mathrm{c}}(\%)$ \\
\hline $\mathbf{1}$ & $\mathrm{NaOCl} / \mathrm{Et}_{3} \mathrm{~N}(0.002 \mathrm{~mol})$ & $\mathrm{CH}_{2} \mathrm{Cl}_{2}$ & $\mathrm{rt}$ & 24 & 50 \\
$\mathbf{2}$ & $\mathrm{NaOCl} / \mathrm{Et}_{3} \mathrm{~N}(0.002 \mathrm{~mol})$ & $\mathrm{CH}_{2} \mathrm{Cl}_{2}$ & 40 & 12 & 55 \\
$\mathbf{3}$ & $\mathrm{I}_{2}(10 \mathrm{~mol} \%)$ & $\mathrm{CH}_{2} \mathrm{Cl}_{2}$ & $\mathrm{rt}$ & 10 & 50 \\
$\mathbf{4}$ & $\mathrm{I}_{2}(10 \mathrm{~mol} \%)$ & $\mathrm{THF}$ & 60 & 10 & 54 \\
$\mathbf{5}$ & $\mathrm{BF}_{3}-\mathrm{Et}_{2} \mathrm{O}(10 \mathrm{~mol} \%)$ & $\mathrm{CH}_{3} \mathrm{CN}$ & 80 & 10 & 55 \\
$\mathbf{6}$ & $\mathrm{CAN}(0.002 \mathrm{~mol})$ & $\mathrm{CH}_{3} \mathrm{CN}$ & 40 & 6 & 60 \\
$\mathbf{7}$ & $\mathrm{Sc}(\mathrm{OTf})_{3}(10 \mathrm{~mol} \%)$ & $\mathrm{CH}_{3} \mathrm{CN}$ & 80 & 10 & 52 \\
$\mathbf{8}$ & No catalyst & $\mathrm{THF}$ & 60 & 10 & 10 \\
$\mathbf{9}$ & No catalyst & $\mathrm{THF}$ & 80 & 10 & 12 \\
$\mathbf{1 0}$ & No catalyst & $\mathrm{CH}_{3} \mathrm{CN}$ & 80 & 10 & 14 \\
\hline
\end{tabular}

${ }^{a}$ catalyst, ${ }^{b}$ solvent used in the reaction and ${ }^{c}$ isolated yields of $3 a$.

With a view to increase the yields of products $3 a-h$ and to reduce reaction time compare to conventional method, we have performed intramolecular cycloaddition using CAN (0.002 mol) as an oxidizing agent under microwave irradiation by taking substrate $2 \mathbf{a}$ as model compound and screened with various solvents. Interestingly in the microwave medium, afforded product $3 a$ in higher yields $(90 \%)$ in short reaction time in acetonitrile solvent compared to conventional heating (Table-2). 
Table 2. Optimization reactions for synthesis compound $3 a$ using CAN (0.002 mol) in conventional and microwave conditions

\begin{tabular}{ccccccccc}
\hline \multicolumn{4}{c}{ Conventional } & \multicolumn{5}{c}{ Microwave } \\
\hline Entry & Solvent & Temp $\left({ }^{\circ} \mathrm{C}\right)$ & Time(h) & Yield (\%) & Solvent & Temp $\left({ }^{\circ} \mathrm{C}\right)$ & Time $(\mathrm{m})$ & Yield (\%) \\
\hline 1 & $\mathrm{CH}_{2} \mathrm{Cl}_{2}$ & 40 & 6 & 50 & $\mathrm{CH}_{2} \mathrm{Cl}_{2}$ & 40 & 10 & 70 \\
2 & $\mathrm{THF}$ & 60 & 6 & 54 & $\mathrm{THF}$ & 60 & 10 & 74 \\
3 & $\mathrm{CH}_{3} \mathrm{CN}$ & 40 & 6 & 60 & $\mathrm{CH}_{3} \mathrm{CN}$ & 40 & 10 & 90 \\
4 & $\mathrm{DMF}$ & 80 & 6 & 56 & $\mathrm{DMF}$ & 60 & 10 & 78 \\
5 & $\mathrm{H}_{2} \mathrm{O}$ & 80 & 6 & 20 & $\mathrm{H}_{2} \mathrm{O}$ & 60 & 10 & 40 \\
\hline
\end{tabular}

The increase in temperature and higher oxidant loading did not improve the yield of product 3a. The reaction furnished very low yields of the product $3 a$ under the oxidant free conditions. The formation of low yields of products probably due to aireal oxidation. After the optimization of microwave assisted reaction conditions, several pyrano isoxazoline chromone derivatives 3a-h prepared in higher yields in short time compare to conventional method. (Table 3) All the synthesized compounds structures were established by spectral analysis. In the ${ }^{1} \mathrm{H} \mathrm{NMR}\left(400 \mathrm{MHz}, \mathrm{CDCl}_{3}\right)$ of compound 3 a newly formed dihydropyranoisoxazoline signals appeared at $\delta: 4.75(\mathrm{~m}, 2 \mathrm{H}, \mathrm{H}-4), 4.2(\mathrm{~m}, 1 \mathrm{H}, \mathrm{H}-3 \mathrm{a}), 3.9-4.1(\mathrm{~m}, 2 \mathrm{H}, \mathrm{H}-3)$ and ${ }^{13} \mathrm{C} \mathrm{NMR}\left(100 M H z, \mathrm{CDCl}_{3}\right)$ signals resonated at $\delta$ : $154.2(\mathrm{C}-12 \mathrm{a}), 70.5(\mathrm{C}-4), 69.2(\mathrm{C}-3), 46.8(\mathrm{C}-3 \mathrm{a})$.

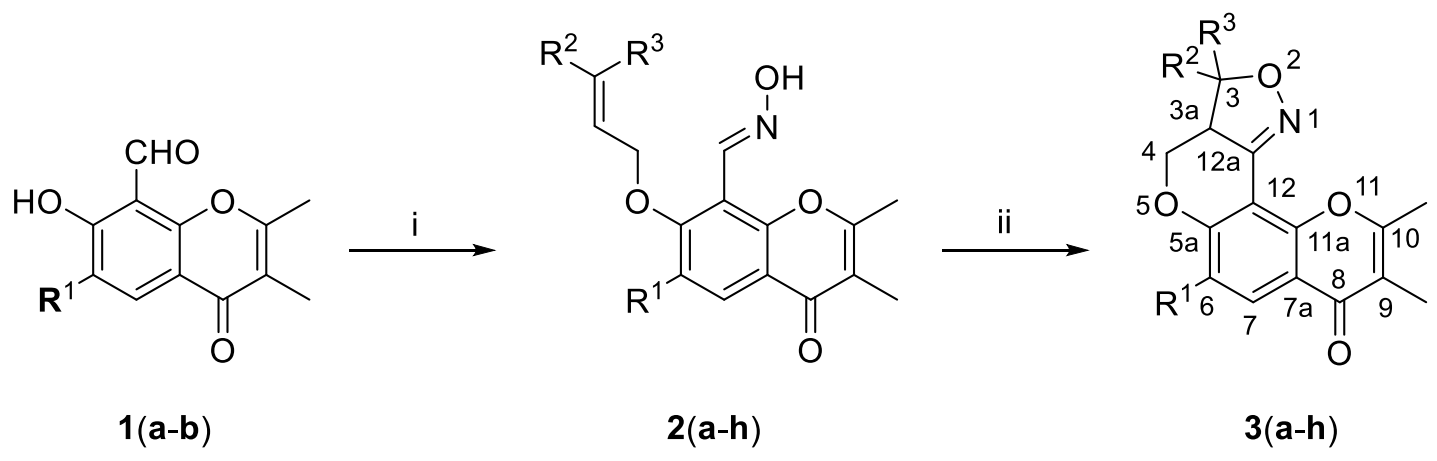

$$
\begin{aligned}
& \text { 1a; } R^{1}=H \quad 2 a ; 3 a ; R^{1}=H, R^{2}=H, R^{3}=H \quad 2 e ; 3 e ; R^{1}=C l, R^{2}=H, R^{3}=H \\
& \text { 1b; } \mathrm{R}^{1}=\mathrm{Cl} \quad \text { 2b; 3b; } \mathrm{R}^{1}=\mathrm{H}, \mathrm{R}^{2}=\mathrm{CH}_{3}, \mathrm{R}^{3}=\mathrm{H} \quad \text { 2f; } \mathbf{3 f} ; \mathrm{R}^{1}=\mathrm{Cl}, \mathrm{R}^{2}=\mathrm{CH}_{3}, \mathrm{R}^{3}=\mathrm{H} \\
& \text { 2c; 3c; } \mathrm{R}^{1}=\mathrm{H}, \mathrm{R}^{2}=\mathrm{CH}_{3}, \mathrm{R}^{3}=\mathrm{CH}_{3} \quad \mathbf{2 g} ; \mathbf{3 g} ; \mathrm{R}^{1}=\mathrm{Cl}, \mathrm{R}^{2}=\mathrm{CH}_{3}, \mathrm{R}^{3}=\mathrm{CH}_{3} \\
& \text { 2d; 3d; } R^{1}=H, R^{2}=P h, R^{3}=H \quad 2 h ; 3 h ; R^{1}=C l, R^{2}=P h, R^{3}=H
\end{aligned}
$$

Scheme 1. Synthesis of pyrano[4,3-c]isaxazoline annulated chromone derivatives (3a-h). Reagents and conditions: (i) (a) Substituted allyl bromides, $\mathrm{K}_{2} \mathrm{CO}_{3}, \mathrm{DMF}, 70{ }^{\circ} \mathrm{C}, 2 \mathrm{~h}$; (b) $\mathrm{CH}_{3} \mathrm{COONa}, \mathrm{NH}_{2} \mathrm{OH} . \mathrm{HCl}, \mathrm{rt}, 1 \mathrm{~h}$; (ii) CAN (20 mol\%), $\mathrm{CH}_{3} \mathrm{CN}, 40{ }^{\circ} \mathrm{C}, \mathrm{MW}, 10 \mathrm{~min}$. 
Table 3. Substrate scope and yields of compounds 3a-h using CAN (0.002 mol) in acetonitrile at $40{ }^{\circ} \mathrm{C}$ under conventional and microwave conditions

\begin{tabular}{cccccc}
\hline \multicolumn{3}{c}{ Conventional } & \multicolumn{2}{c}{ Microwave } \\
\hline Entry & Product & Time (h) & Yield (\%) & Time (m) & Yield (\%) \\
\hline 1 & 3a & 6 & 60 & 10 & 89 \\
2 & 3b & 6 & 52 & 10 & 94 \\
3 & 3c & 6 & 50 & 10 & 92 \\
4 & 3d & 6 & 48 & 10 & 88 \\
5 & 3e & 6 & 48 & 10 & 88 \\
6 & 3f & 6 & 52 & 10 & 90 \\
7 & 3g & 6 & 50 & 10 & 90 \\
8 & 3h & 6 & 48 & 10 & 87 \\
\hline
\end{tabular}

Encouraged by these results, we next planned for the synthesis of diverse bicyclic pyrano isoxazole annulated chromones having high substitution. Similar to the preparation of compounds $\mathbf{2 a - h}$ as discussed above, 7propargyloxychromone-8-aldoximes 4a-f were prepared in one pot by coupling 7-hydroxy-8-formylchromone with propargyl bromide and hydroxyl amine hydrochloride in alkaline sodium acetate in DMF. The intermediates 4a-f were subjected to in situ generated nitrile oxide 1,3-dipolar cycloaddition at alkyne under conventional as well as microwave conditions using optimized CAN (0.002 mol) as oxidant in acetonitrile solvent to afford pyrano isoxazole fused chromone derivatives. The microwave irradiation furnished the products 5a-f in good yields (84-91\%) compare to conventional method (Scheme-2). The reaction conditions and yields of products summarized in Table-4. The structures of all the compounds 5a-f confirmed by spectral analysis. The ${ }^{1} \mathrm{H} \mathrm{NMR}\left(400 \mathrm{MHz}, \mathrm{CDCl}_{3}\right)$ of compound 5 a newly formed dihydropyrano isoxazole signals appeared at $\delta: 8.30(\mathrm{~s}, 1 \mathrm{H}, \mathrm{H}-3) 5.40(\mathrm{~s}, 2 \mathrm{H}, \mathrm{H}-4)$, and ${ }^{13} \mathrm{C} \mathrm{NMR}\left(100 \mathrm{MHz}, \mathrm{CDCl}_{3}\right)$ signals resonated at $\delta$ : 153.6(C-12a), 110.2(C-3), 103.9(C-3a), 62.3(C-4).

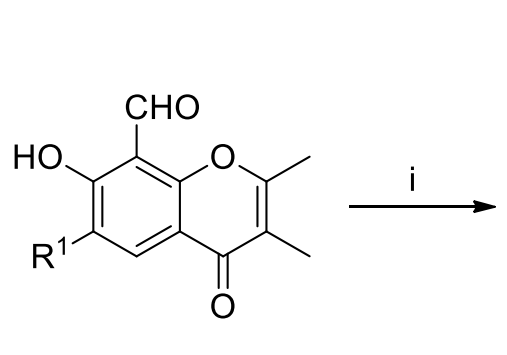

$1(a-b)$

$$
\begin{aligned}
& \text { 1a; } R^{1}=H \\
& \text { 2b; } R^{1}=C l
\end{aligned}
$$

$\mathrm{R}^{4}$

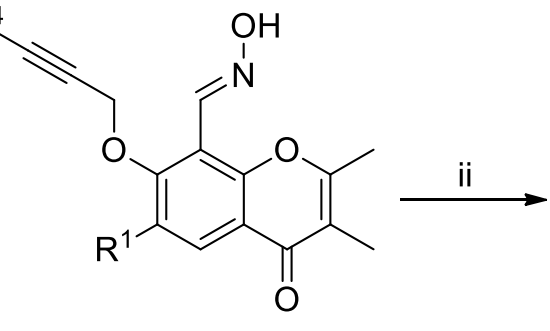

4(a-f)

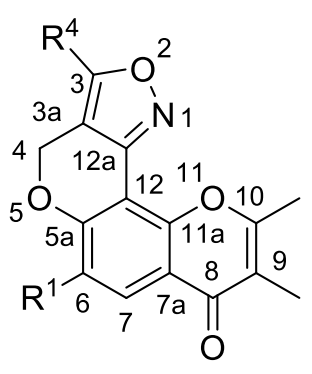

5(a-f)

2. Synthesis of pyrano $[4,3-c]$ isoxazole annulated chromone derivatives (5a-f). Reagents and conditions: i) (a) Substituted propargyl bromides, $\mathrm{K}_{2} \mathrm{CO}_{3}, \mathrm{DMF}, 70{ }^{\circ} \mathrm{C}, 2 \mathrm{~h}$; (b) $\mathrm{CH}_{3} \mathrm{COONa}, \mathrm{NH}_{2} \mathrm{OH} . \mathrm{HCl}, \mathrm{rt}, 1 \mathrm{~h}$; ii) CAN (20 mol\%), $\mathrm{CH}_{3} \mathrm{CN}, 40{ }^{\circ} \mathrm{C}, \mathrm{MW}, 15 \mathrm{~min}$. 
Table 4. Substrate scope and yields of compounds 5a-f using CAN (0.002 mol) in acetonitrile at $40{ }^{\circ} \mathrm{C}$ under conventional and microwave heating

\begin{tabular}{cccccc}
\hline \multicolumn{3}{c}{ Conventional } & & \multicolumn{2}{c}{ Microwave } \\
\hline Entry & Product & Time (h) & Yield (\%) & Time (m) & Yield (\%) \\
\hline 1 & 5a & 6 & 62 & 15 & 86 \\
2 & 5b & 6 & 64 & 15 & 88 \\
3 & 5c & 6 & 65 & 15 & 91 \\
4 & $\mathbf{5 d}$ & 6 & 62 & 15 & 90 \\
5 & $\mathbf{5 e}$ & 6 & 60 & 15 & 86 \\
6 & $\mathbf{5 f}$ & 6 & 62 & 15 & 84 \\
\hline
\end{tabular}

\section{Conclusions}

In conclusion, we developed a simple and efficient protocol for the synthesis of highly substituted 6-6-6-5membered tetracyclic pyrano isoxazoline/isoxazole annulated chromone derivatives 3a-h and 5a-f from allyloxy and propargyloxy appended chromone aldoxime derivatives $\mathbf{2 a - h}$ and $\mathbf{4 a - f}$ using ceric ammonium nitrate (CAN) under conventional and microwave assisted, regioselective intramolecular 1,3-dipolar cycloaddition. The key step of synthetic route is the one pot generation of 7-alkyloxy-8-aldoxime chromones. We obtained higher yields of products $\mathbf{3 a - h}$ and $\mathbf{5 a - f}$ in eco-friendly microwave irradiation compared to conventional heating. We believe that these newly developed chromone based isoxazoline/isoxazole scaffolds will find diverse applications in chemical biology and medicinal chemistry.

\section{Experimental Section}

General. Silica gel (60-120 mesh) for column chromatography was purchased from M/s Acme Synthetic Chemicals (Mumbai, India) and pre-coated TLC plates (Silica gel 60F254) were purchased from Merck (Darmstadt, Germany). All the chemicals, reagents and solvents were purchased from M/s SD Fine Chemicals (Mumbai, India) with highest grade of purity. Microwave reactions were performed in a Multi synth series microwave system (Milestone). The ${ }^{1} \mathrm{H}$ NMR and ${ }^{13} \mathrm{C}$ NMR spectra were recorded on a Bruker spectrometer at 400 and $100 \mathrm{MHz}$ and TMS used as an internal standard. Chemical shifts relative to TMS as internal standards were given as $\delta$ values in ppm. Mass spectra were recorded using electron spray ionization on Waters e2695 Separators module (Waters, Milford, MA, USA) mass spectrometer. IR spectra were recorded on a Fourier transform (FT-IR), USA (Perkin-Elmer model 337) instrument. The melting points were determined on a Barnstead Electro Thermal 9200 Instrument.

\section{General procedure for the synthesis of 2,3-dimethyl-7-0-allylated-8-aldoxime chromones (2a-h)}

To the stirred solution of compounds $1 \mathbf{a}-\mathbf{b}(1.0 \mathrm{mmol})$ and potassium carbonate $(0.2 \mathrm{mmol}) \mathrm{in} \mathrm{DMF}(10 \mathrm{~mL})$ allyl bromides $(1.2 \mathrm{mmol})$ were added and the reaction mixture was stirred at $70{ }^{\circ} \mathrm{C}$ for $2 \mathrm{~h}$, then reaction mixture was cooled to $\mathrm{rt}$ and added sodium acetate $(3.63 \mathrm{mmol})$, Hydroxylamine hydrochloride $(1.0 \mathrm{mmol})$ to the mixture and stirred for $1 \mathrm{~h}$. After completion of reaction pale yellow colour solids appeared which were 
poured in water $(20 \mathrm{~mL})$ the solid precipitate was collected by filtration, washed with water and dried at $50{ }^{\circ} \mathrm{C}$ to afford $2 \mathrm{a}-\mathrm{h}$ as white solids with good yields (70-90\%).

7-(allyloxy)-2,3-dimethyl-4-oxo-4H-chromene-8-carbaldehyde oxime (2a) mp 202-205 ${ }^{\circ} \mathrm{C}$, Yield 85\%. IR vmax, $\mathrm{cm}^{-1}: 1650(\mathrm{C}=\mathrm{N}), 1685\left(\mathrm{CO}\right.$, ketone). ${ }^{1} \mathrm{H} \mathrm{NMR}\left(400 \mathrm{MHz}, \mathrm{CDCl}_{3}\right) \delta: 8.30(\mathrm{~s}, 1 \mathrm{H}), 7.72(\mathrm{~d}, J 8.8 \mathrm{~Hz}, 1 \mathrm{H}), 6.95(\mathrm{~d}, J$ $8.8 \mathrm{~Hz}, 1 \mathrm{H}), 6.01(\mathrm{~m}, 1 \mathrm{H}), 5.41(\mathrm{~m}, 1 \mathrm{H}), 5.25(\mathrm{~m}, 1 \mathrm{H}), 4.50-4.80(\mathrm{~m}, 2 \mathrm{H}), 2.43(\mathrm{~s}, 3 \mathrm{H}), 2.21(\mathrm{~s}, 3 \mathrm{H}) .{ }^{13} \mathrm{CNMR}(100$ $\mathrm{MHz}) \delta: 183.0,158.5,156.1,142.2,133.2,130.1,118.0,116.9,116.1,113.5,112.2,107.5,70.2,14.2,9.0$. ESIHRMS $m / z$ calcd for $\mathrm{C}_{15} \mathrm{H}_{15} \mathrm{NO}_{4} 274.1079[\mathrm{M}+\mathrm{H}]^{+}$, found 274.1034 .

General procedure for the synthesis of 9,10-dimethyl-3a,4-dihydropyrano[2',3':5,6]chromeno[4,3-c]isoxazol8(3H)-ones 3(a-h)

Conventional. To the stirred solution of compound $2 \mathrm{a}-\mathrm{h}(1.0 \mathrm{mmol})$ in acetonitrile $(10 \mathrm{~mL})$ was added ceric ammonium nitrate (CAN) $(2 \mathrm{mmol})$ at $0{ }^{\circ} \mathrm{C}$ then the reaction mixture was stirred for $6 \mathrm{~h}$ at $40{ }^{\circ} \mathrm{C}$. The reaction progress was monitored by TLC, after completion of the reaction, $20 \mathrm{~mL}$ of water was added and extracted with chloroform and washed with brine solution. The crude material was purified by column chromatography in chloroform/methanol (9:1) to give products $3 \mathbf{a}-\mathbf{h}$.

Microwave. To the stirred solution of compounds $2 \mathrm{a}-\mathbf{h}(1.0 \mathrm{mmol})$ in acetonitrile $(10 \mathrm{~mL})$ was added ceric ammonium nitrate (CAN) $(2 \mathrm{mmol})$ at $0{ }^{\circ} \mathrm{C}$ then the reaction mixture was placed in a quartz tube inserted into a screw capped Teflon vial and subjected to microwave irradiation $\left(200 \mathrm{~W}\right.$ ) for $10 \mathrm{~min}$, at $40{ }^{\circ} \mathrm{C}$ after completion of the reaction (monitored by $\mathrm{TLC}$ ), $50 \mathrm{~mL}$ of cold water was added to reaction mixture and extracted with chloroform, washed with brine solution. The crude sample purified by column chromatography eluting with chloroform/methanol (9:1) in excellent yields.

9,10-Dimethyl-3a,4-dihydropyran[2',3':5,6]chromeno[4,3-c]isoxazol-8(3H)-one (3a) mp $219-222{ }^{\circ} \mathrm{C}, \quad$ Yield 89\%. IR spectrum, v, cm ${ }^{-1}: 1630(\mathrm{C}=0) .{ }^{1} \mathrm{H}$ NMR $\left(400 \mathrm{MHz}, \mathrm{CDCl}_{3}\right) \delta: 8.15(\mathrm{~d}, J 8.65 \mathrm{~Hz}, 1 \mathrm{H}), 6.95(\mathrm{~d}, J 8.8 \mathrm{~Hz}$, $1 \mathrm{H}), 4.75(\mathrm{~m}, 2 \mathrm{H}), 4.20(\mathrm{~m}, 1 \mathrm{H}), 3.90-4.10(\mathrm{~m}, 2 \mathrm{H}), 2.45(\mathrm{~s}, 3 \mathrm{H}), 2.05(\mathrm{~s}, 3 \mathrm{H}) .{ }^{13} \mathrm{C} \mathrm{NMR}\left(100 \mathrm{MHz}^{\mathrm{CDCl}}\right)_{3} \delta:$ $176.9,162.3,159.5,154.2,149.8,129.5,118.4,117.4,114.5,102.3,70.5,69.2,46.8,18.3,10.1$. ESI-HRMS m/z calcd for $\mathrm{C}_{15} \mathrm{H}_{13} \mathrm{NO}_{4}$. $272.0923[\mathrm{M}+\mathrm{H}]^{+}$, found 272.0914 .

3,9,10-Trimethyl-3a,4-dihydropyrano[2',3':5,6]chromeno[4,3-c]isoxazol-8(3H)-one (3b) mp 231-233 ${ }^{\circ} \mathrm{C}$, Yield 94\%. IR spectrum, v, cm ${ }^{-1}: 1632(\mathrm{C}=\mathrm{O}) .{ }^{1} \mathrm{H} N M R\left(400 \mathrm{MHz}_{\mathrm{CDCl}}\right) \delta: 8.15(\mathrm{~d}, \mathrm{~J} 8.5 \mathrm{~Hz}, 1 \mathrm{H}), 6.95(\mathrm{~d}, \mathrm{~J} 8.8 \mathrm{~Hz}, 1 \mathrm{H})$, $4.40(\mathrm{~m}, 1 \mathrm{H}), 4.70(\mathrm{~m}, 1 \mathrm{H}), 4.20(\mathrm{~m}, 1 \mathrm{H}), 3.60(\mathrm{~m}, 1 \mathrm{H}), 2.45(\mathrm{~s}, 3 \mathrm{H}), 2.05(\mathrm{~s}, 3 \mathrm{H}), 1.60(\mathrm{~s}, 3 \mathrm{H}) .{ }^{13} \mathrm{C} \mathrm{NMR}(100$ $\mathrm{MHz}_{1} \mathrm{CDCl}_{3}$ ) $\delta: 181.0,161.6,160.2,155.6,153.1,130.9,126.2,118.5,115.0,108.5,73.0,70.6,44.2,21.5,18.3$, 10.2. ESI-HRMS $m / z$ calcd for $\mathrm{C}_{16} \mathrm{H}_{15} \mathrm{NO}_{4} 286.1079[\mathrm{M}+\mathrm{H}]^{+}$, found 286.1070

3,3,9,10-Tetramethyl-3a,4-dihydropyrano[2',3':5,6]chromeno[4,3-c]isoxazol-8(3H)-one (3c) $\mathrm{mp} 213-216{ }^{\circ} \mathrm{C}$,

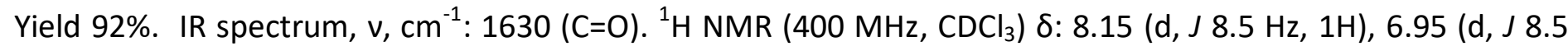
$\mathrm{Hz}, 1 \mathrm{H}$ ), 4.60 (dd, J 1.0, 8.5 Hz, 1H), 4.15 (dd, J 1.0, $8.5 \mathrm{~Hz}, 1 \mathrm{H}), 3.50$ (dd, J 1.0, 8.5Hz, 1H), 2.50 (s, $3 \mathrm{H}$ ), 2.05 (s, $3 \mathrm{H}), 1.65(\mathrm{~s}, 3 \mathrm{H}), 1.25(\mathrm{~s}, 3 \mathrm{H}) .{ }^{13} \mathrm{C} N M R\left(100 \mathrm{MHz}, \mathrm{CDCl}_{3}\right) \delta: 180.0,164.8,162.6,155.1,130.8,125.8,120.4$, $119.5,115.6,111.2,71.0,41.3,25.5,25.1,18.6,10.0,8.0$. ESI-HRMS $m / z$ calcd for $\mathrm{C}_{17} \mathrm{H}_{17} \mathrm{NO}_{4} 300.1236[\mathrm{M}+\mathrm{H}]^{+}$, found 300.1230 .

9,10-Dimethyl-3-phenyl-3a,4-dihydropyrano[2',3':5,6]chromeno[4,3-c]isoxazol-8(3H)-one (3d) mp 218-221 ${ }^{\circ} \mathrm{C}$, Yield 88\%. IR spectrum, v, $\mathrm{cm}^{-1}: 1626(\mathrm{C}=0) .{ }^{1} \mathrm{H} \mathrm{NMR}\left(400 \mathrm{MHz}, \mathrm{CDCl}_{3}\right) \delta: 8.15(\mathrm{~d}, J 8.8 \mathrm{~Hz}, 1 \mathrm{H}), 7.40-7.60$ $(\mathrm{m}, 6 \mathrm{H}), 6.95(\mathrm{~d}, J 8.6 \mathrm{~Hz}, 1 \mathrm{H}), 5.30(\mathrm{~d}, J 8.8 \mathrm{~Hz}, 1 \mathrm{H}), 4.85(\mathrm{~m}, 1 \mathrm{H}), 4.35(\mathrm{~m}, 1 \mathrm{H}), 4.00(\mathrm{~m}, 1 \mathrm{H}), 2.50(\mathrm{~s}, 3 \mathrm{H}), 2.05$ (s, 3H). ${ }^{13} \mathrm{C}$ NMR $\left(100 \mathrm{MHz}, \mathrm{CDCl}_{3}\right) \delta: 179.1,165.6,163.2,160.8,156.3,155.0,140.1,132.5,130.4,129.1$, $128.5,127.8,126.6,125.8,120.4,119.6,113.8,85.6,72.1,41.6,18.3,10.2$. ESI-HRMS $m / z$ calcd for $\mathrm{C}_{21} \mathrm{H}_{17} \mathrm{NO}_{4}$ $348.1236[\mathrm{M}+\mathrm{H}]^{+}$, found 348.1235 . 
6-Chloro-9,10-dimethyl-3a,4-dihydropyrano[2',3':5,6]chromeno[4,3-c]isoxazol-8(3H)-one (3e) $\mathrm{mp} 236-239$ ${ }^{\circ} \mathrm{C}$, Yield 88\%. IR spectrum, v, $\mathrm{cm}^{-1}: 1621$ (C=O). ${ }^{1} \mathrm{H}$ NMR (400 MHz, $\left.\mathrm{CDCl}_{3}\right) \delta: 2.05(\mathrm{~s}, 3 \mathrm{H}), 2.45(\mathrm{~s}, 3 \mathrm{H}), 3.90-$ $4.10(\mathrm{~m}, 2 \mathrm{H}), 4.25(\mathrm{~m}, 1 \mathrm{H}), 4.75(\mathrm{~m}, 1 \mathrm{H}), 4.95(\mathrm{~m}, 1 \mathrm{H}), 8.25(\mathrm{~s}, 1 \mathrm{H}) .{ }^{13} \mathrm{C} \mathrm{NMR}\left(100 \mathrm{MHz}, \mathrm{CDCl}_{3}\right) \delta: 10.2,18.0$, 46.6, 69.5, 70.4, 102.8, 117.6, 118.4, 122.5, 125.3, 149.3, 154.2, 159.8, 162.4, 176.8. ESI-MS: $\mathrm{m} / \mathrm{z} 306$ [M+H]

6-Chloro-3,9,10-trimethyl-3a,4-dihydropyrano[2',3':5,6]chromeno[4,3-c]isoxazol-8(3H)-one (3f) $\mathrm{mp} 240-242$

${ }^{\circ} \mathrm{C}$, Yield 90\%. IR spectrum, v, $\mathrm{cm}^{-1}: 1629$ (C=O). ${ }^{1} \mathrm{H}$ NMR $\left(400 \mathrm{MHz}, \mathrm{CDCl}_{3}\right) \delta: 8.25(\mathrm{~s}, 1 \mathrm{H}), 4.70(\mathrm{~m}, 1 \mathrm{H}), 4.40$ $(\mathrm{m}, 1 \mathrm{H}), 4.20(\mathrm{~m}, 1 \mathrm{H}), 3.60(\mathrm{~m}, 1 \mathrm{H}), 2.45(\mathrm{~s}, 3 \mathrm{H}), 2.05(\mathrm{~s}, 3 \mathrm{H}), 1.60(\mathrm{~s}, 3 \mathrm{H}) .{ }^{13} \mathrm{C} \mathrm{NMR}\left(100 \mathrm{MHz}, \mathrm{CDCl}_{3}\right) \delta: 180.1$, $163.8,160.5,156.1,155.3,131.3,125.6,122.5,115.8,108.4,73.6,70.8,44.6,21.2,18.9,10.9$. ESI-MS: $\mathrm{m} / \mathrm{z} 320$ $[\mathrm{M}+\mathrm{H}]^{+}$.

6-Chloro-3,3,9,10-tetramethyl-3a,4-dihydropyrano[2',3':5,6]chromeno[4,3-c]isoxazol-8(3H)-one (3g) mp 231-

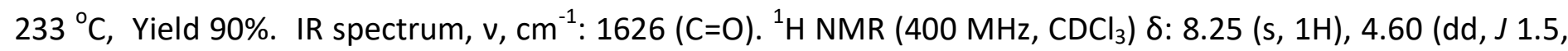
$8.5 \mathrm{~Hz}, 1 \mathrm{H}), 4.15$ (dd, J 1.0, $8.5 \mathrm{~Hz}, 1 \mathrm{H}$ ), 3.50 (dd, J 1.0, $8.5 \mathrm{~Hz}, 1 \mathrm{H}), 2.50(\mathrm{~s}, 3 \mathrm{H}), 2.05$ (s, 3H), 1.65 (s, $3 \mathrm{H}), 1.25$ (s, 3H). ${ }^{13} \mathrm{C}$ NMR $\left(100 \mathrm{MHz}_{\mathrm{CDCl}}\right.$ ) $\delta: 179.6,163.7,161.1,156.3,129.4,126.3,125.5,118.6,114.1,110.0,84.3$, 70.4, 40.3, 25.5, 25.1, 18.8, 10.2. $\mathrm{C}_{17} \mathrm{H}_{16} \mathrm{CINO}_{4}$. ESI-HRMS $\mathrm{m} / z$ calcd for $333.0846[\mathrm{M}+\mathrm{H}]^{+}$, found 333.0760.

6-Chloro-9,10-dimethyl-3-phenyl-3a,4-dihydropyrano[2',3':5,6]chromeno[4,3-c]isoxazol-8(3H)-one (3h) $\mathrm{mp}$ 218-221 ${ }^{\circ} \mathrm{C}$, Yield 87\%. IR spectrum, v, $\mathrm{cm}^{-1}: 1635$ (C=O). ${ }^{1} \mathrm{H}$ NMR (400 MHz, $\left.\mathrm{CDCl}_{3}\right)$ $\delta: 8.25(\mathrm{~s}, 1 \mathrm{H}), 7.40-7.60$ $(\mathrm{m}, 5 \mathrm{H}), 4.85(\mathrm{~m}, 1 \mathrm{H}), 4.35(\mathrm{~m}, 1 \mathrm{H}), 4.00(\mathrm{~m}, 1 \mathrm{H}), 2.50(\mathrm{~s}, 3 \mathrm{H}), 2.05(\mathrm{~s}, 3 \mathrm{H}) .{ }^{13} \mathrm{C} \mathrm{NMR}\left(100 \mathrm{MHz}, \mathrm{CDCl}_{3}\right) \delta: 180.0$, 164.6, 163.7, 162.3, 158.2, 157.2, 142.1, 137.8, 134.5, 132.6, 130.8, 128.4, 125.8, 124.3, 120.4, 122.6, 112.1, 87.3, 70.8, 40.2, 18.5, 10.6. ESI-MS: $m / z 382[\mathrm{M}+\mathrm{H}]^{+}$.

\section{General procedure for the synthesis 9,10-dimethyl-7-0-propargylated-8-aldoxime chromones (4a-f)}

Propargyl bromides $(1.2 \mathrm{mmol})$ were added to the stirred solution of compound $1 \mathbf{a}-\mathbf{b}(1.0 \mathrm{mmol})$ and potassium carbonate $(0.2 \mathrm{mmol})$ in DMF $(10 \mathrm{~mL})$ and the reaction mixture was stirred at $70{ }^{\circ} \mathrm{C}$ for $2 \mathrm{~h}$, after completion of the reaction indicated by TLC, the reaction mixture was cooled to RT then Sodium acetate (1.0 $\mathrm{mmol}$ ) and Hydroxylamine hydrochloride $(1.0 \mathrm{mmol})$ was added to the reaction mixture and stirred for $1 \mathrm{~h}$. After completion of reaction pale yellow colour solid was appeared which was poured in water $(20 \mathrm{~mL})$, the solid precipitate was collected by filtration, washed with water and dried at $50{ }^{\circ} \mathrm{C}$ to afford $4 a-f$ as white solids with good yields (75-91\%).

2,3-Dimethyl-4-oxo-7-(prop-2-yl-1-yloxy)-4H-chromene-8-carbaldehyde oxime (4a) mp 206-210 ${ }^{\circ} \mathrm{C}$, Yield 85\%. IR spectrum, v, $\mathrm{cm}^{-1}: 1655(\mathrm{C}=\mathrm{N}) .{ }^{1} \mathrm{H}$ NMR $\left(400 \mathrm{MHz}, \mathrm{CDCl}_{3}\right) \delta: 8.31(\mathrm{~s}, 1 \mathrm{H}), 7.75(\mathrm{~d}, J 8.7 \mathrm{~Hz}, 1 \mathrm{H}), 6.80(\mathrm{~d}, J$ $8.7 \mathrm{~Hz}, 1 \mathrm{H}), 4.68(\mathrm{~s}, 1 \mathrm{H}), 3.32(\mathrm{~s}, 1 \mathrm{H}), 2.42(\mathrm{~s}, 3 \mathrm{H}), 2.20(\mathrm{~s}, 3 \mathrm{H}) .{ }^{13} \mathrm{C} \mathrm{NMR}\left(100 \mathrm{MHz}, \mathrm{CDCl}_{3}\right) \delta: 182.5,166.5$, $158.5,156.3,142.5,130.1,116.5,113.8,112.4,107.5,78.9,76.2,56.5,14.5,8.9$. ESI-HRMS $\mathrm{m} / \mathrm{z}$ calcd for $\mathrm{C}_{15} \mathrm{H}_{13} \mathrm{NO}_{4} 272.0923[\mathrm{M}+\mathrm{H}]^{+}$, found 272.0878

General procedure for the Synthesis of 9,10-dimethylpyrano[2',3':5,6]chromeno[4,3-c]isoxazol-8(4H)-ones 5(a-f)

Conventional. To the stirred solution of compound $4 a-f(1 \mathrm{mmol})$ in acetonitrile $(10 \mathrm{~mL})$ was added ceric ammonium nitrate (CAN) $(2 \mathrm{mmol})$ at $0{ }^{\circ} \mathrm{C}$ then the reaction mixture was stirred for $6 \mathrm{~h}$ at $40{ }^{\circ} \mathrm{C}$. The reaction progress was monitored by TLC, after completion of the reaction; $20 \mathrm{~mL}$ of water was added to reaction mixture to get solid precipitate it was collected by filtration, washed with water and dried at $50{ }^{\circ} \mathrm{C}$ to afford final product $\mathbf{5 a - f}$ as white solid with good yields.

Microwave. To the stirred solution of compounds $4 a-f(1.0 \mathrm{mmol})$ in acetonitrile $(10 \mathrm{~mL})$ was added ceric ammonium nitrate (CAN) $(2 \mathrm{mmol})$ at $0{ }^{\circ} \mathrm{C}$ then the reaction mixture was placed in a quartz tube inserted into a screw capped Teflon vial and subjected to microwave irradiation ( $200 \mathrm{~W}$ ) for 15 min the progress of reaction 
monitored by TLC, after completion of the reaction, $50 \mathrm{~mL}$ of cold water mixture was added to reaction mixture. The crude sample purified through column chromatography (hexane/ethyl acetate 4:1) to yield products 5 a-f (white solids).

9,10-Dimethylpyrano[2',3':5,6]chromeno[4,3-c]isoxazol-8(4H)-one (5a) mp 229-231 ${ }^{\circ} \mathrm{C}$, Yield 86\%. IR spectrum, v, $\mathrm{cm}^{-1}: 1636(\mathrm{C}=0) .{ }^{1} \mathrm{H} \mathrm{NMR}\left(400 \mathrm{MHz}, \mathrm{CDCl}_{3}\right) \delta: 8.30(\mathrm{~s}, 1 \mathrm{H}), 8.20(\mathrm{~d}, J 8.8 \mathrm{~Hz}, 1 \mathrm{H}), 7.00(\mathrm{~d}, J 8.8 \mathrm{~Hz}$, $1 \mathrm{H}), 5.40(\mathrm{~s}, 2 \mathrm{H}), 2.55(\mathrm{~s}, 3 \mathrm{H}), 2.05(\mathrm{~s}, 3 \mathrm{H}) .{ }^{13} \mathrm{C} \mathrm{NMR}\left(100 \mathrm{MHz}, \mathrm{CDCl}_{3}\right) \delta: 176.9,162.5,159.6,153.6,151.8$, 150.2, 129.6, 118.8, 117.6, 115.4, 110.2, 103.9, 62.3, 18.5, 10.4. ESI-HRMS m/z calcd for $\mathrm{C}_{15} \mathrm{H}_{11} \mathrm{NO}_{4} 270.0766$ $[\mathrm{M}+\mathrm{H}]^{+}$, found 270.0757 .

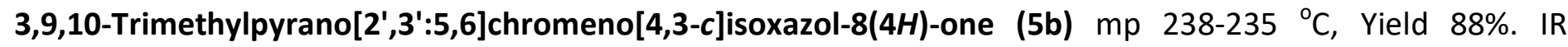
spectrum, v, $\mathrm{cm}^{-1}: 1626(\mathrm{C}=0) .{ }^{1} \mathrm{H} \mathrm{NMR}\left(400 \mathrm{MHz}, \mathrm{CDCl}_{3}\right) \delta: 8.20(\mathrm{~d}, J 8.8 \mathrm{~Hz}, 1 \mathrm{H}), 7.00(\mathrm{~d}, J 8.8 \mathrm{~Hz}, 1 \mathrm{H}), 5.40(\mathrm{~s}$, $2 \mathrm{H}), 2.75(\mathrm{~s}, 3 \mathrm{H}), 2.55(\mathrm{~s}, 3 \mathrm{H}), 2.05(\mathrm{~s}, 3 \mathrm{H}) .{ }^{13} \mathrm{C} \mathrm{NMR}\left(100 \mathrm{MHz}, \mathrm{CDCl}_{3}\right) \delta: 178.8,162.6,160.2,155.6,153.7$, $152.9,130.5,120.3,117.8,116.5,111.4,102.6,61.2,18.5,14.3,10.6$. ESI-MS: $m / z 284[\mathrm{M}+\mathrm{H}]^{+}$.

3-Ethyl-9,10-dimethylpyrano[2',3':5,6]chromeno[4,3-c]isoxazol-8(4H)-one (5c) mp 209-212 ${ }^{\circ} \mathrm{C}$, Yield 91\%. IR spectrum, v, $\mathrm{cm}^{-1}: 1622$ (C=O). ${ }^{1} \mathrm{H}$ NMR $\left(400 \mathrm{MHz}, \mathrm{CDCl}_{3}\right) \delta: 1.12$ (t, J $\left.7.5 \mathrm{~Hz}, 3 \mathrm{H}\right), 2.05(\mathrm{~s}, 3 \mathrm{H}), 2.25(\mathrm{q}, J 7.5 \mathrm{~Hz}$, $2 \mathrm{H}), 2.55(\mathrm{~s}, 3 \mathrm{H}), 4.90(\mathrm{~s}, 2 \mathrm{H}), 7.20(\mathrm{~d}, J 8.8 \mathrm{~Hz}, 1 \mathrm{H}), 8.41(\mathrm{~d}, J 8.8 \mathrm{~Hz}, 1 \mathrm{H}) .{ }^{13} \mathrm{C} \mathrm{NMR}\left(100 \mathrm{MHz}, \mathrm{CDCl}_{3}\right) \delta: 180.1$, $175.4,160.5,158.2,156.8,155.2,132.6,130.3,129.6,120.6,118.6,114.5,65.6,23.5,20.3,18.8,10.2$. ESIHRMS $m / z$ calcd for $\mathrm{C}_{17} \mathrm{H}_{15} \mathrm{NO}_{4} 298.1079[\mathrm{M}+\mathrm{H}]^{+}$, found 298.1070

6-chloro-9,10-dimethylpyrano[2',3':5,6]chromeno[4,3-c]isoxazol-8(4H)-one (5d) mp 249-251 ${ }^{\circ} \mathrm{C}$, Yield $90 \%$. IR spectrum, v, cm ${ }^{-1}: 1630$ (C=O). ${ }^{1} \mathrm{H}$ NMR (400 MHz, $\mathrm{CDCl}_{3}$ ) $\delta: 8.30$ (s, 1H), 8.20 (s, $1 \mathrm{H}$ ), 5.40 (s, $\left.2 \mathrm{H}\right), 2.55$ (s, $3 \mathrm{H}), 2.05$ (s, 3H). ${ }^{13} \mathrm{C} \mathrm{NMR}\left(100 \mathrm{MHz}, \mathrm{CDCl}_{3}\right) \delta: 179.1,162.8,160.6,155.8,152.6,151.2,130.9,125.4,120.8$, $117.6,111.2,100.8,62.3,18.2,9.9$. ESI-MS: $m / z 304[\mathrm{M}+\mathrm{H}]^{+}$.

6-chloro-3,9,10-trimethylpyrano[2',3':5,6]chromeno[4,3-c]isoxazol-8(4H)-one (5e) mp 248-249 ${ }^{\circ} \mathrm{C}$, Yield 86\%. IR spectrum, v, cm ${ }^{-1}: 1628$ (C=0). ${ }^{1} \mathrm{H}$ NMR (400 MHz, CDCl $)$ $\delta: 8.20(\mathrm{~s}, 1 \mathrm{H}), 7.00(\mathrm{~d}, J 8.5 \mathrm{~Hz}, 1 \mathrm{H}), 5.40(\mathrm{~s}, 2 \mathrm{H})$, $2.75(\mathrm{~s}, 3 \mathrm{H}), 2.55$ (s, 3H), 2.05 (s, 3H). ${ }^{13} \mathrm{C} \mathrm{NMR}\left(100 \mathrm{MHz}, \mathrm{CDCl}_{3}\right) \delta: 176.8,162.5,159.2,153.6,151.2,150.3$, $129.4,118.6,117.2,115.0,110.1,103.9,62.3,18.4,14.3,10.9$. ESI-MS: $m / z 318[\mathrm{M}+\mathrm{H}]^{+}$.

6-chloro-3-ethyl-9,10-dimethylpyrano[2',3':5,6]chromeno[4,3-c]isoxazol-8(4H)-one (5f) mp $220-221{ }^{\circ} \mathrm{C}$, Yield 84\%. IR spectrum, v, cm ${ }^{-1}: 1626$ (C=O). ${ }^{1} \mathrm{H}$ NMR (400 MHz, $\left.\mathrm{CDCl}_{3}\right) \delta: 8.24(\mathrm{~s}, 1 \mathrm{H}), 7.20(\mathrm{~d}, J 7.8 \mathrm{~Hz}, 1 \mathrm{H}), 4.90(\mathrm{~s}$, $2 \mathrm{H}), 2.55(\mathrm{~s}, 3 \mathrm{H}), 2.25(\mathrm{q}, J 7.2 \mathrm{~Hz}, 2 \mathrm{H}),, 2.05(\mathrm{~s}, 3 \mathrm{H}), 1.12(\mathrm{t}, J 7.2 \mathrm{~Hz}, 3 \mathrm{H}) .{ }^{13} \mathrm{C} \mathrm{NMR}\left(100 \mathrm{MHz}, \mathrm{CDCl}_{3}\right) \delta: 10.0$, $18.2,20.1,25.3,65.8,120.0,122.3,125.4,130.2,132.5,135.4,154.1,155.6,158.6,165.1,170.1,180.4$. ESI$\mathrm{MS}: \mathrm{m} / \mathrm{z} 332[\mathrm{M}+\mathrm{H}]^{+}$.

\section{Acknowledgements}

SPN thanks to University Grants Commission (UGC), New Delhi, India for provided senior research fellowship and authors are thankful to Department of Chemistry, Central Facilities for Research and Development (CFRD), Osmania University, Hyderabad, India for providing Laboratory and analysis facilities.

\section{Supplementary Material}

The experimental procedures and IR, ${ }^{1} \mathrm{H}$ NMR and ${ }^{13} \mathrm{C}$ NMR spectra for compounds $\mathbf{3 a - d}, \mathbf{3 g}, \mathbf{5 a}$ and $\mathbf{5 c}$ associated with this article are available as supplementary data in the online version of the text. 


\section{References}

1. Houghton, P. J.; Osibogun, I. M.; Woldemariam, T. Z.; Jones, K. Planta Med. 1995, 61, 154. https://doi.org/10.1055/s-2006-958037

2. Tezuka, Y.; Gewali, M. B.; Ali, M. S.; Banskota, A. H.; Kadota, S. J. Nat. Prod. 2001, 64, 208. https://doi.org/10.1021/np000492t

3. Gaspar, A.; Matos, M. J.; Garrido, J.; Uriarte, E.; Borges, F. Chem. Rev. 2014, 114(9), 4960. https://doi.org/10.1021/cr400265z

4. Rangappa, S.K.; Srinivasa, B.; Ranjith, K. P.; Geetha, R. B. Eur. J. Med. Chem. 2014, 78, 340.

5. Reis, J.; Gaspar, A.; Milhazes, N.; Borges, F. J. Med. Chem. 2017, 60, 7941. https://doi.org/10.1021/acs.jmedchem.6b01720

6. Silva, C. F. M.; Diana C. G. A. P.; Silva, A. M. S. Chem. Med. Chem. 2016, 11(20), 2252. https://doi.org/10.1002/cmdc.201600359

7. Silva, C. F. M.; Batista, V. F.; Diana, C. G. A. P.; Silva. A. M. S. Expert opinion in drug discovery, 2018, 13(9), 795.

https://doi.org/10.1080/17460441.2018.1494720

8. Abu-Bakr, S. M.; Khidre, M. D.; Omar, M. A.; Samira, A. S.; Awad, H. M. J. Het. Chem. 2020, 57(2), 731. https://doi.org/10.1002/jhet.3813

9. Jayaprakash, R. Y.; Pravardhan, R. E.; Sridhar, B.; Lakshmi, J. K.; SubbaReddy, B. V. Tetrahedron Lett. 2016, 57, 2853. https://doi.org/10.1016/j.tetlet.2016.05.055

10. Cindy, C.; Broeder. Curr. Org. Synthesis. 2011, 8, 628.

11. Yamamoto, T.; Fujita, K.; Asari, S.; Chiba, A.; Kataba, Y.; Ohsumi, K.; Ohmuta, N.; lida, Y.; Ijichi, C.; Iwayama, S.; Fukuchi, N.; Shoji, M. Bioorg. Med. Chem. Lett. 2007, 17, 3736. https://doi.org/10.1016/j.bmcl.2007.04.024

12. Kuzmin, V. E.; Artemenko, A. G.; Muratov, E. N.; Volineckaya, I. L.; Makarov, V. A.; Riabova, O. B.; Wutzler, P.; Schmidtke, M. J. Med Chem. 2007, 50, 4205. https://doi.org/10.1021/jm0704806

13. Soni, A. K.; Krupadanam, G. L. D.; Srimannarayana, G. Arkivoc. 2006, 16, 35. https://doi.org/10.3998/ark.5550190.0007.g04

14. Kaffy, J.; Pontikis, R.; Carrez, D.; Croisy, A.; claude, M.; Jean-Claude, F. Bioorg. Med. Chem. 2006, $14,4067$. https://doi.org/10.1016/j.bmc.2006.02.001

15. Rowley, M.; Broughton, H. B.; Collins, I.; Baker, R.; Emms, F.; Marwood, R.; Patel, S.; Ragan, C. I. J. Med. Chem. 1996, 39, 1943.

16. Frolund, B.; Jorgensen, A. T.; Tagmose, L.; Stensbol, T. B.; Vestergaard, H. T.; Engblom, C.; Kristiansen, U.; Sanchez, C.; KrogsgaardLarsen, P.; Liljefors, T. J. Med. Chem. 2002, 45, 2454. https://doi.org/10.1021/im0200270

17. Talley, J. J. Prog. Med. Chem. 1999, 13, 201. https://doi.org/10.1080/02668739900700161

18. Talley, J. J.; Brown, D. L.; Carter, J. S.; Graneto, M. J.; Koboldt, C. M.; Masferrer, J. L.; Perkins, W. E.; Rogers, R. S.; Shaffer, A. F.; Zhang, Y. Y.; Zweifel, B. S.; Seibert, K. J. Med. Chem. 2000, 43, 775. https://doi.org/10.1080/02668739900700161 
19. Li, W.-T.; Hwang, D.-R.; Chen, C.-P.; Shen, C.-W.; Huang, C.-L.; Chen, T.-W.; Lin, C.-H.; Chang, Y.-L.; Chang, Y.-Y.; Lo, Y.-K.; Tseng, H.-Y.; Lin, C.-C.; Song, J.-S.; Chen, H.-C.; Chen, S.-J.; Wu, S.-H.; Chen ,C.T. J. Med. Chem. 2003, 46, 1706. https://doi.org/10.1021/jm020471r

20. Jie, Z.; Jun, M; Lin, H. -Z.; Chen, Y.; Peng, H. S. Bioorg. Med. Chem. 2018, 26(12), 3065.

21. Bustosa, C.; Elies, M.; Carcamo, J. -G.; Aguilar, M. N.; Sancheza, C.; Ignacio, M.-V.; Hiroyuki, N.; Angela, M. -S.; Zaratee, X.; Schott, E. New J. Chem. 2015, 39(6), 4295. https://doi.org/10.1039/C4NJ02427C

22. Pinho, E. O.; Melo, T. M. V. D. Curr. Org. Chem. 2005, 9, 925. https://doi.org/10.2174/1385272054368420

23. Gothelf, K.V.; Jorgensen, K, A. Chem. Rev. 1998, 98, 863. https://doi.org/10.1021/cr970324e

24. Mustafa, J. R.; Veerababurao, K.; Chun-Wei. K.; Raju, B. R.; Ching-Fa, Y. Green Chem. 2010, $12,1090$. https://doi.org/10.1039/b926085d

25. Robert, E.; Sammelson, T. M.; Luis, J.; Galietta, V.; Verkman, A. S.; Mark, J. K. Bioorg. Med. Chem. Lett. 2003, 13, 2509. https://doi.org/10.1016/S0960-894X(03)00482-7

26. Jonathan, T. D.; Martin, T. R.; Kauhaluoma, J. Y.; Anita, D.; Wentworth, B. Z.; Wentworth, J.; Kim, D. J. J. Org. Chem. 2005, 70, 7810. https://doi.org/10.1021/j0050410b

27. Brindabon, R.; Rajendra, N. D. Monatsh. Chem. 2010, 141, 763. https://doi.org/10.1007/s00706-010-0323-3

28. Gonçalves, R. S. B.; Dos Santos, M.; Bernadat, G. ; Bonnet-Delpont, D. ; Crousse, B. Beilstein J. Org. Chem. 2003, 9, 2387

https://doi.org/10.3762/bjoc.9.275

29. Quan, C.; Kurth, M. J. Org. Chem. 2004, 69, 1470. https://doi.org/10.1021/jo0352124

30. Lidstrom, P.; Tierney, J.; Wathey, B.; Westman, J. Tetrahedron 2001, 57(45), 9225, https://doi.org/10.1016/S0040-4020(01)00906-1

31. Jayapraksh, R. Y.; Krupadanam, G. L. D. Indian J. Chem. 2000, 39B, 610. 